\title{
A retrospective study evaluating the correlation between pelvic bone marrow dose and acute hematological toxicity in cervical cancer patients treated with concurrent chemoradiation
}

\author{
Sahil Grover ${ }^{1}$, Himanshu Joshi ${ }^{1}$, Prekshi Chaudhary ${ }^{2}$, Dinesh Singh ${ }^{3}$ \\ 1 DNB Resident, Radiation Oncology, Max Super Speciality Hospital, Vaishali, India, ${ }^{2}$ Consultant, Radiation Oncology, Max Super Speciality Hospital, \\ Vaishali, India, ${ }^{3}$ Director, Radiation Oncology, Max Super Speciality Hospital, Vaishali, India \\ Keywords: bone marrow, dosimetric parameters, acute hematological toxicity, cervical cancer \\ https://doi.org/10.53876/001c.28426
}

International Journal of Cancer Care and Delivery

Vol. 1, Issue Supplement 1, 2021

\section{BACKGROUND:}

Concurrent chemoradiation followed by brachytherapy is the standard of care for locally advanced cervical cancer. However, this treatment is associated with a significant risk of hematological toxicity, which in turn may result in infection, canceled chemotherapy cycles or suboptimal chemo dose intensity and may increase overall treatment time, thereby leading to poorer prognosis. All these factors may be associated with a higher probability of treatment failure. Knowing the fact that pelvic bone is the primary site of hematopoiesis, we examined the correlation between pelvic bone marrow dose/volume parameters and acute hematological toxicity incidence in a cohort of cervical cancer patients, receiving definitive chemoradiation followed by brachytherapy.

\section{METHOD:}

Medical records of all the patients treated by concurrent chemoradiotherapy from January 2019 to December 2020 were reviewed. Baseline and weekly hematological parameters were collected. Bone marrow was retrospectively delineated and bone marrow volumes $(\mathrm{V})$ receiving 5,10,20,30,40 Gy (V5, V10, V20, V30, V40 respectively) were calculated. Logistic regression was used to analyze association between hematological toxicity and dose volume histogram parameters.

\section{RESULT:}

A total of 30 patients were enrolled for statistical analysis. We observed reduction in hemoglobin, TLC, Absolute lymphocyte count from their baseline value. Hematological toxicity CTCAE version 3.0 grade $2,3,4+$ seen in many patients. Anemia with grade 2 toxicity was more than grade 3 (26.6\% vs $10 \%)$, the same happened with leukopenia ( $26.6 \%$ grade 2 vs $3.3 \%$ grade 3 ) whereas lymphopenia grade 3 were seen in more patients than those with grade 2 (46.6\% vs $30 \%$ ) with grade 4 lymphopenia also seen in 1 patient. When all these parameters nadir mean were compared with their baseline mean values, they showed a significant difference with a $\mathrm{p}$ value of $<0.001$ in all of them. It was also observed that V5 pelvic marrow mean value of $95.3 \pm 2.72$ showed toxicity grade $<2$ and $96.8 \pm 1.73$ showed hematological grade $\geqslant 2$ with a significant difference (p- 0.04 ), such significant difference also seen with V20 pelvic bone marrow dose of $73 \pm 4.24$ and $75.2 \pm 2.94(p-0.05)$.

\section{CONCLUSIONS:}

The IMRT reduced the volume of bone marrow irradiated at higher doses and so the incidence of acute hematological toxicity in cervical cancer patients undergoing concurrent chemoradiotherapy. The following dose constraints could be proposed to decrease risk of hematological toxicity: V5<95\%, V20<69\%, V40<30\%. 